\title{
Physical mechanism for the mechanical reinforcement in nanotube-polymer composite materials
}

\author{
A. Wall, J. N. Coleman, and M. S. Ferreira \\ Physics Department, Trinity College Dublin, Dublin 2, Ireland \\ (Received 16 August 2004; revised manuscript received 13 October 2004; published 25 March 2005)
}

\begin{abstract}
The significant increase in the Young's modulus of nanotube-polymer composites is often correlated with the formation of a crystalline layer of polymers surrounding the nanotubes. Although the improved stress transfer between the stiffer nanotube and the softer polymer matrix has been attributed to the presence of this wellordered polymeric layer, the actual mechanism for this stress transfer is still unclear. To clarify this matter, we model the polymer-nanotube composite by harmonic chains interacting with a rigid periodic potential, an extension of the so-called Frenkel-Kontorova model. We identify the origin of the reinforcement with the occurrence of a templating transition, in which polymers are constrained by the periodic potential of the underlying nanotube. The model is potentially capable of predicting the ideal combination of polymers and nanotube diameters to maximally enhance the mechanical properties of composite structures.
\end{abstract}

DOI: 10.1103/PhysRevB.71.125421

PACS number(s): $62.25 .+\mathrm{g}$

More than a decade after the discovery of carbon nanotubes, these nanoscale cylindrical structures are still the subject of intensive scientific research due to their superlative physical properties. Mechanically very strong and good conductors of thermal and electronic currents, it is not surprising that nanotubes are excellent fillers in polymeric composite materials and can substantially improve their mechanical, electronic, and thermal responses. ${ }^{1-7}$ Concerning the mechanical properties, nanotube/polymer composites provide order-of-magnitude increases in strength and stiffness of thin films when compared to typical carbon-fiber/polymer composites. In the form of fibers, these composite materials display strength and toughness superior to steel and Kevlar, respectively. ${ }^{1}$ The fibers are easy to weave and sew and, due to their high electronic capacitance, have promising electronic-textile applications, the most remarkable being the possibility of manufacturing mechanically resistant fabrics with energy-storing capability.

Stress-strain measurements in nanotube-polymer composite films find that their Young's modulus can be increased by a factor 4 when less than $1 \%$ of nanotubes are added to the polymer matrix. ${ }^{8}$ The appearance of large values of reinforcement with such a minute amount of nanotubes is directly correlated with the formation of a crystalline layer of polymers surrounding the immediate vicinity of the nanotube surface. Further evidence for this correlation is given by the fact that similar nanotubes in the presence of noncrystalline polymers display significantly lower levels of reinforcement. ${ }^{2,3,8}$ Concerning the morphology of this crystalline layer, experimental observations at low coverage show that polymers tend to coil at well-defined angles to the nanotube axis. This is consistent with molecular dynamics simulations $^{9}$ and agrees with the mathematical concept that strings constrained within a cylindrical geometry are optimally packed in a helical orientation. ${ }^{10}$ In fact, a recent macroscopic model describing the basic mechanisms for this type of crystallization points to a range of preferential coiling angles that polymers may follow when coating the walls of a nanotube. ${ }^{11}$

Despite the experimental evidence, there is no convincing theoretical support for the correlation between crystallinity and mechanical reinforcement in polymer-nanotube composites. Based on purely macroscopic arguments, the physical origin of the mechanical reinforcement is commonly attributed to the improved load transfer that results from the formation of a crystalline coating surrounding the nanotubes. This assumes that the rigidity of the embedded carbon nanotubes can only be probed in the composite structure when the stress is efficiently transferred across the nanotube-polymer interface. Although this is certainly a relevant factor, it is not sufficient to provide a quantitative description of reinforcement. This is confirmed by the fact that the so-called rule of mixtures (RM), a traditional method for calculating the mechanical response of macroscopic composite structures, overestimates the Young's modulus of nanotube-polymer composites significantly when the known nanotube modulus is used as an input parameter. ${ }^{2,12}$ This failure results from the erroneous assumption of full stress transfer between the different constituent phases of the composite material. Without a way of quantifying the stress transfer across the nanotubepolymer interface we cannot reproduce the observed levels of reinforcement in a reliable manner. This does not seem possible in a simple macroscopic formalism and calls for an alternative approach that involves the microscopic structure of the component parts.

With such a motivation, in this paper we propose a simple model that is able to bridge this gap between the microscopic and macroscopic scales, elucidating the origin of the stress transfer across the nanotube-polymer interface. As a result, the physical mechanism for the enhanced mechanical response in composite materials is clarified and more importantly, the model points to a nonmonotonic reinforcement dependence on the nanotube diameter. Such a nonmonotonic behavior is remarkable and indicates the possibility of predicting the adequate combination of materials to tailor and optimize the mechanical reinforcement in such structures. Our claims are given additional support by the reasonably good agreement between our results and available experimental data.

We start by modelling the polymer-coated nanotubes as infinitely long cylinders of radius $r$ wrapped around by semiflexible strands. Since we are interested in the stress transfer 
a)

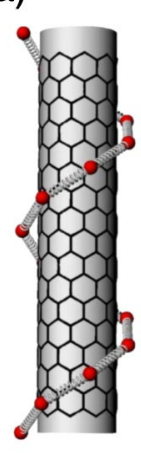

b)

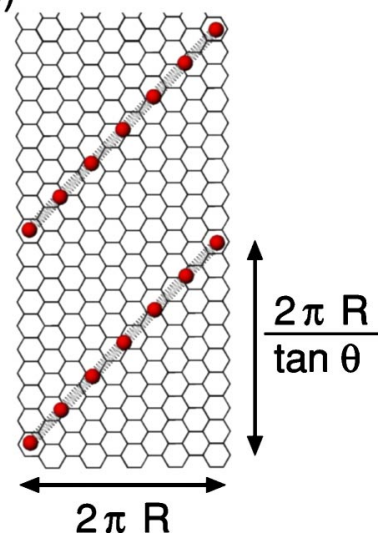

FIG. 1. (Color online) (a) Schematic representation of a single polymer strand coiling along the nanotube axis at an angle $\theta$. The strand is represented by monomers connected by elastic springs. (b) In the two-dimensional depiction, the unwrapped tube is represented by a stripe of width $2 \pi R$ and the coiling angle $\theta$ defines a unit cell of length $2 \pi R / \tan \theta$.

across the polymer-nanotube interface, it is sufficient to study this transfer for a single monolayer of polymer coating. The polymer strands are represented by equally spaced monomers separated by harmonic springs of natural length $\ell_{0}$ and lie on a cylindrical surface of radius $R=r+\delta$, where $\delta$ is the van der Waals distance to the tube surface, hereafter considered to be constant $(\delta=3.5 \AA)$. Following experimental evidence, ${ }^{13}$ we assume that the polymers coil around the nanotube surface at a well-defined angle $\theta$ relative to the axial direction, as schematically shown for a single strand in Fig. 1. The atomic structure of the nanotube is depicted by the hexagonal lattice both on the cylindrical wall [Fig. 1(a)] and on the flat surface that results from unwrapping the nanotube [Fig. 1(b)].

To address the issue of stress transfer in crystalline composite materials, we must establish how the stretching of nanotube-coated polymer strands compares with that of nanotube-free polymers. Young's modulus is the most representative quantity to be studied and is defined as the slope of the stress-strain curve. We must then find how the stressinduced strain experienced by a polymer strand is affected by the presence of the hexagonal potential produced by the underlying nanotube lattice. A suitable model that accounts for the effect of this potential is the so-called Frenkel-Kontorova model, traditionally used to describe adsorbed monolayers on atomically flat surfaces. ${ }^{14-18}$ This one-dimensional model consists of a linear chain of particles harmonically coupled by springs of elastic constant $k$ and natural length $\ell_{0}$ subject to an external sinusoidal potential of period $\lambda$ and amplitude $U$. The Hamiltonian for the particles is commonly written as

$$
H=\sum_{j}\left\{\frac{k}{2}\left(X_{j}-X_{j-1}-\ell_{0}\right)^{2}+V\left(X_{j}\right)\right\},
$$

where $X_{j}$ represents the position of particle $j$ and in the case of the standard Frenkel-Kontorova model $V(x)=U[1$ $-\cos (2 \pi x / \lambda)]$. The ground state features of this model have

been extensively studied in which the static equilibrium configuration of the system results from the interplay of the two characteristic length scales $\left(\ell_{0}\right.$ and $\left.\lambda\right)$, the confining potential and the resistance to stretching. It is obvious that the natural monodisperse distribution of interparticle distances of an isolated chain is altered by the presence of the external potential, but despite local fluctuations of these distances, the total length of the chain can be fully described by the average interparticle separation, hereafter defined as $\omega=\left\langle X_{j}-X_{j-1}\right\rangle$. This quantity can be evaluated by finding the ground-state configuration of the system, for which we use the method of effective potentials due to Griffiths and Chou. ${ }^{18}$ This method provides a computationally efficient way of obtaining the equilibrium positions for the particles $\left(X_{j}\right)$ subjected to the external periodic potential, from which the value of $\omega$ can be derived.

Since we are interested in the mechanical response of the chain in the presence of the periodic potential, we need to investigate how the value of $\omega$ depends on the stretching force. The effect of this force can be mapped into an increase of the natural bond length $\ell_{0}$, which makes the function $\omega\left(\ell_{0}\right)$ the relevant quantity to be investigated. Two distinct scenarios are identified, depending on whether or not the resulting particle distribution is commensurate with the periodic potential. For the case of incommensurate distribution, it has been shown that the ground state value for $\omega$ can be infinitesimally shifted by a gradual increase of the bond length $\ell_{0} \cdot{ }^{18}$ This means that despite small rearrangements of the particles, the average particle distance increases linearly with the length $\ell_{0}$ and should reflect the intrinsic stiffness of the atomic chain. In the commensurate case, on the other hand, the particles are trapped by the minima of the external potential. In this way, there is a range of values of $\ell_{0}$ for which the average separation $\omega$ does not change. This corresponds to the case in which the chain follows the same periodicity as the external potential and will be referred to as being templated by the potential. This can be seen in Fig. 2(a) where the average equilibrium interparticle distance $\omega$ of an atomic chain is plotted as a function of the bond length $\ell_{0}$ for the sinusoidal potential depicted in the inset. Plateaus in the $\omega\left(\ell_{0}\right)$ curve corresponding to the commensurate regime are surrounded by linearly varying regions that characterize the incommensurate case. Also known as Devil's staircase, ${ }^{19}$ this diagram provides information about the templating capacity of the potential. Bearing in mind that the chain ultimately represents a polymer strand and that the potential is the result of the interaction with the nanotube lattice, in the templated regime the strand displays not its intrinsic elastic response but that associated with the underlying nanotube. In other words, the plateaus of the Devil's staircase represent the situation in which the polymers experience the significantly superior stiffness of the nanotube. Therefore, by identifying the conditions for the appearance of these plateaus in the corresponding Devil's staircase, one can in principle control the reinforcement levels in a composite material. Although this argument was based on results for one-dimensional chains in the presence of sinusoidal potentials, it can be easily extended to the case of polymer strands on the walls of a nanotube. 

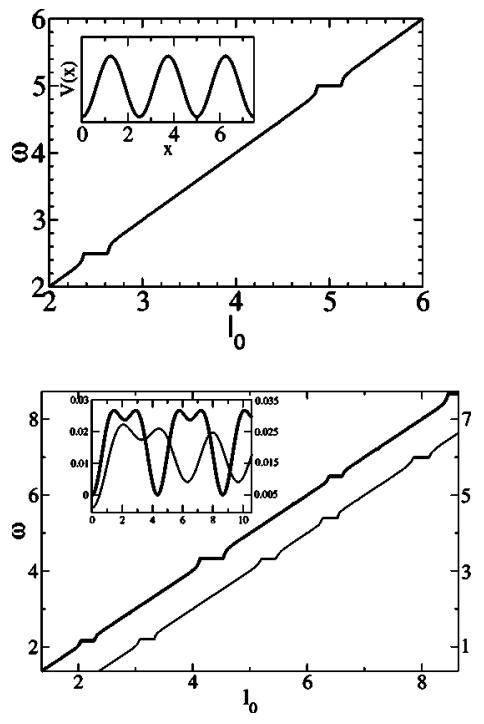

FIG. 2. (a) Average particle separation $\omega$ as a function of the natural bond length $\ell_{0}$ displaying a typical Devil's staircase found in the Frenkel-Kontorova model. Both quantities are expressed in similar arbitrary units. The commensurate phases represented by the plateaus are surrounded by linearly varying sections characterizing the incommensurate ones. The inset shows the sinusoidal potential (in equivalent units) generating the staircase. (b) Similar Devil's staircases (now expressed in $\AA$ ) obtained for strands coiling around a zigzag nanotube $(5,0)$ (lattice parameter $a=2.45 \AA$ ) at different coiling angles $\left(\theta=0^{\circ}\right.$ for the thick line and $\theta=74^{\circ}$ for the thin line). The inset shows the corresponding potentials. Thick (thin) lines are read on the left (right) vertical axis.

In the case of polymer strands coiling around the nanotube walls, it is convenient to visualize the polymer strands on top of the unwrapped nanotubes, as seen in Fig. 1(b). Depending on the coiling angle, rather than simple sinusoidal potentials the interaction with the hexagonally symmetric nanotube atoms generates periodic potentials with more than one Fourier component, the amplitude of which ( $U$ $=30 \mathrm{meV})$ is found in previously reported potential corrugations. ${ }^{20}$ In this case, the effective potential experienced by the polymer strands has more local minima within a given period $\lambda$. Such a higher density of local minima is likely to enhance the density of commensurate phases and consequently the templating capacity of the potential. In fact, this is confirmed by Fig. 2(b), which shows two distinct Devil's staircases associated with their respective potentials (shown in the inset). Both the staircases and the potentials have been displaced along the vertical axis for the sake of distinction but each individual curve corresponds to a different coiling angle. Since the appearance of plateaus indicate that strands are being templated by the underlying periodic potential, it is important to calculate the frequency with which these plateaus appear for a given type of nanotube. By averaging over all possible coiling angles, we define a templating fraction $T_{f}$ that gives the percentage of polymer strands experiencing the stiffness of the nanotube. In this way, rather than assuming that all crystalline polymers surrounding the nanotube display perfect stress transfer, as implied by the standard rule-of-mixtures, we can quantify the fraction of cases for which this optimal transfer takes place.

Besides the nanotube- and crystalline-polymer phases, an additional region of amorphous polymer embeds the other two in the composite material. When calculating the elastic properties of these composites, one must account for the contributions from all those three phases. For the Young's modulus, for instance, each phase will have both a volumetric and a surface-area contribution, the former giving the intrinsic modulus of the phase in question, and the latter describing the interaction between neighboring phases. Bearing in mind that we are mainly interested in the stress transfer across the polymer-nanotube interface, we can disregard the volumetric contribution from the crystalline phase and focus only on the effect that a crystalline monolayer brings to the interaction with the other two regions. Since the amorphous phase is entangled with polymers at the edge of the crystalline layer we assume a perfect load transfer between these two phases. Concerning the interaction with the nanotubes, the templating fraction $T_{f}$ gives the percentage of crystalline interfacial layers with optimal stress transfer. We can then express the Young's modulus $Y_{C}$ of the composite as $Y_{C}=T_{f} V_{f} Y_{N} \eta+\left(1-V_{f}\right) Y_{P}$, where $Y_{N}$ and $Y_{P}$ are the Young's modulus of the nanotube and the polymer, respectively, $V_{f}$ is the fractional volume taken by the nanotube and the coefficient $\eta=3 / 8$ accounts for the random distribution of nanotubes in the plane of the film. ${ }^{8}$ It is worth mentioning that the only difference to the usual RM expression for the Young's modulus of composite structures is in the introduction of the templating fraction $T_{f}$ in the first term on the right-hand side of the equation for $Y_{C}$. It is evident from the expression above that the larger the templating fraction, the larger the Young's modulus of the composite structure, which means that a reliable way of calculating the templating fraction is important to provide a good estimate for the mechanical reinforcement induced by nanotubes.

For a given polymer with a well-defined bond length $\ell_{0}$, the obvious way of calculating $T_{f}$ consists of selecting a large number of coiling angles $\theta$, obtain the ground state configuration for each individual angle and count the fraction of cases for which the polymer lies inside a templating plateaux. This so-called counting-by-inspection procedure is slow and computationally intensive since reliable results for $T_{f}$ requires a large number of angles. It is evident from Fig. 2(b) that the occurrence of plateaus in the staircases correlates with the existence of a high concentration of local minima, which suggests that there might be a way of obtaining the templating fraction by associating it with the distribution of those minima. In fact, analysis of a number of Devil's staircase graphs yields a geometric interpretation that simplifies the counting procedure. It consists of projecting the hexagonal lattice of a nanotube of radius $r$ onto a cylinder of radius $r+\delta$. Simple geometry shows that while distances in the axial direction are unchanged, projected distances in the circumferential direction are scaled by a factor $(1+\delta / r)$. The plateaus do not occur randomly but are correlated with the positions of the potential minima of this projected lattice. More precisely, the plateaus appear every time a polymer atom lies in the proximity of a potential minimum, that is, in the proximity of an edge of the projected lattice. How close the polymer atoms must be to the local minima 

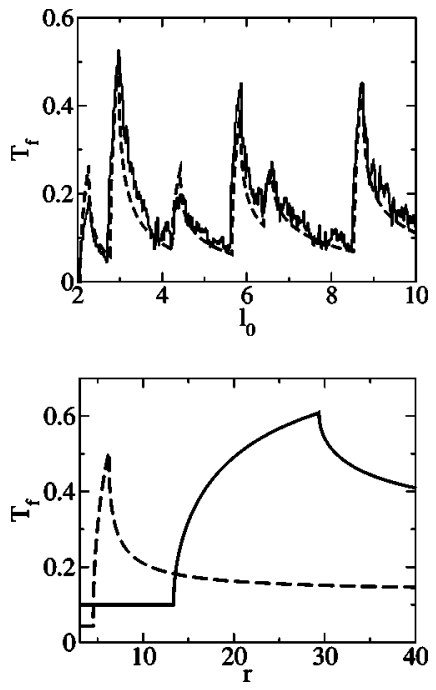

FIG. 3. (a) Templating fraction $T_{f}$ as a function of the bond length $\ell_{0}$ (in $\AA$ ). The full line diplays $T_{f}$ calculated by averaging over a large number of possible coiling angles and counting the fraction of cases in which a given value of $\ell_{0}$ lies inside a plateaux. The dashed line is obtained by the graphical method (see text). Despite small fluctuations, the agreement is evident. (b) Templating fraction as a function of nanotube radius (zigzag) for two different values of $\ell_{0} \quad\left(\ell_{0}=2.45 \AA\right.$ for the full line and $\ell_{0}=3.0 \AA$ for the dashed line). Elastic constant was chosen to be $k=21 \mathrm{eV} / \AA^{2}$, in accordance with estimated values for polyvinylalcohol.

depends on the width of the corresponding templating plateaux. Therefore, by superimposing the polymer strands (at a given bond length $\ell_{0}$ ) to the projected hexagonal lattice one can graphically check whether or not the former is templated by the latter, a much simpler procedure than the countingby-inspection method. Despite the tremendous simplification, the agreement between the two counting methods is remarkable, as shown in Fig. 3(a), where the templating fraction calculated by both methods is plotted as a function of the bond length $\ell_{0}$. Except for small fluctuations, the solid line representing the counting-by-inspection method agrees with the graphical method represented by the dashed line. The figure shows that the templating fraction reaches periodical maximum values, which indicates that the right choice of polymer parameters might lead to increased levels of reinforcement. Unfortunately, such set of parameters cannot be varied in a continuous fashion due to the limited number of polymer types.

A more controllable quantity that can be continuously varied is the nanotube diameter. By plotting the templating fraction (obtained by the graphical method) as a function of the nanotube radius, we show in Fig. 3(b) that $T_{f}$ also behaves nonmonotonically. Maximum values of the templating fraction at certain diameters predict that with an adequate selection of nanotubes one should be capable of optimizing the level of mechanical reinforcement in polymer-nanotube composites. The figure shows two different types of polymers with distinct optimal diameters suggesting a general trend in the diameter dependence of nanotube-induced reinforcement. This trend indicates that for any given polymer showing

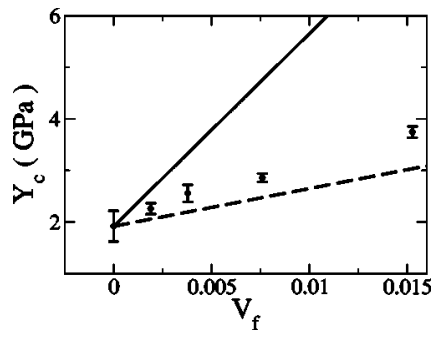

FIG. 4. Young's modulus of a nanotube-polyvinylalcohol composite as a function of the nanotube volume fraction. Points are measured values, the solid line represents the standard RM and the dashed line corresponds to the modified RM with the templating fraction $T_{f}=0.20$, consistent with a nanotube of corresponding diameter. Individual Young's moduli are taken as $Y_{N}=1 \mathrm{TPa}$, $Y_{P}=1.92 \mathrm{GPa}$.

crystallinity in the presence of nanotubes, there is always a suitable diameter that will optimize the mechanical properties of the corresponding composite. Such a nonmonotonic behavior does not appear in a recent experimental study in which the level of reinforcement is measured for a range of nanotube diameters. ${ }^{21}$ However, it is worth stressing that the reported results cover only a very narrow range of small diameters and a slightly wider range of large diameters. The nonmonotonic behavior here predicted lies in the intermediate range of diameters not covered by the aforementioned experiment.

Finally, we can test our model by comparing the estimated value of $Y_{c}$ with experimental data available in the literature. It is important to stress that we have chosen a system with a thin crystalline phase. Thicker crystalline coatings are expected to affect the degree of reinforcement but would require an extension of the present model to include the volumetric contribution of this phase. Figure 4 shows the measured Young's modulus of nanotube-polyvinilalcohol composites as a function of the volume fraction $V_{f}$ of inserted nanotubes. Arc discharge multiwalled nanotubes (diameter $24 \mathrm{~nm}$ ) were blended with polyvinylalcohol with volume fraction $V_{f}$ ranging from $0 \%$ to $0.16 \%$. The full line is the standard RM expression and clearly overestimates the observed levels of reinforcement represented by the points. The dashed line representing our results with the calculated templating fraction $T_{f}=0.20$ for a nanotube of corresponding diameter [obtained from Fig. 3(b)] displays significant improvement in the comparison with the measured values. The disagreement between our results and the observed values can be explained by the fact that a small but finite thickness for the crystalline phase enhances the reinforcement of the system.

In summary, we have presented a model describing how the interaction of crystalline polymers surrounding the vicinity of carbon nanotubes affects the mechanical properties of nanotube-polymer composites. Rather than assuming full stress transfer between the different phases of the composite, the model suggests that only a fraction of the polymer layers are templated to the underlying nanotube lattice. This fraction of templated polymers experiences the significantly larger stiffness of the nanotube and is responsible for the enhancement of the mechanical response of the composite. 
Furthermore, the model predicts that by tuning the nanotube diameter one can optimize the templating fraction for each type of polymeric material, thus maximally enhancing the mechanical properties of the composite. In other words, despite the simplicity of our model the results here presented bring forward an important message, namely, that an appropriate set of polymers and nanotubes diameters can always be combined to achieve maximum reinforcement. In addition to clarifying the reinforcement mechanisms in nanotubepolymer composite materials, we believe that our results can stimulate further simulations with improved predictive power, which in turn will guide the experimental search for stronger light-weight composite materials.

The authors acknowledge financial support from Science Foundation Ireland and IRCSET.
${ }^{1}$ A. B. Dalton, S. Collins, E. Munoz, J. M. Razal, V. H. Ebron, J. P. Ferraris, J. N. Coleman, B. G. Kim, and R. H. Baughman, Nature (London) 423, 703 (2003).

${ }^{2}$ M. Cadek, J. N. Coleman, V. Barron, K. Hedicke, and W. J. Blau, Appl. Phys. Lett. 81, 5123 (2002).

${ }^{3}$ J. K. W. Sandler, S. Pegel, M. Cadek, F. Gojny, M. van Es, J. Lohmar, W. J. Blau, K. Schulte, A. H. Windle, and M. S. P. Shaffer, Polymer 45, 2001 (2004).

${ }^{4}$ B. E. Kilbride, J. N. Coleman, J. Fraysee, P. Fournet, M. Cadek, A. Drury, S. Hutzler, S. Roth, and W. J. Blau, J. Appl. Phys. 92, 4024 (2002).

${ }^{5}$ J. K. W. Sandler, J. E. Kirk, I. A. Kinloch, M. S. P. Shaffer, and A. H. Windle, Polymer 44, 5893 (2003).

${ }^{6}$ M. J. Biercuk, M. C. Llaguno, M. Radosavljevic, J. K. Hyun, J. E. Fischer, and A. T. Johnson, Appl. Phys. Lett. 80, 2767 (2002).

${ }^{7}$ C. Wei, D. Srivastava, and K. Cho, Nano Lett. 2, 647 (2002).

${ }^{8}$ J. N. Coleman, M. Cadek, R. Blake, V. Nicolosi, K. P. Ryan, C. Belton, A. Fonseca, J. B. Nagy, Y. K. Gun'ko, and W. J. Blau, Adv. Funct. Mater. 14, 791 (2004).

${ }^{9}$ M. Zheng, A. Jagota, E. D. Semke, B. A. Diner, R. S. McLean, S. R. Lustig, R. E. Richardson, and N. G. Tassi, Nat. Mater. 2, 338 (2003).

${ }^{10}$ A. Maritan, C. Micheletti, A. Trovato, and J. R. Banavar, Nature
(London) 406, 287 (2000).

${ }^{11}$ J. N. Coleman and M. S. Ferreira, Appl. Phys. Lett. 84, 798 (2004).

${ }^{12}$ M. S. P. Shaffer and A. H. Windle, Adv. Mater. (Weinheim, Ger.) 11, 937 (1999).

${ }^{13}$ B. McCarthy, J. N. Coleman, R. Czerw, A. B. Dalton, M. Panhuis, A. Maiti, A. Drury, P. Bernier, J. Nagy, B. Lahr, H. J. Byrne, D. L. Carroll, and W. J. Blau, J. Phys. Chem. B 106, 3087 (2002).

${ }^{14}$ M. Weiss and F. J. Elmer, Phys. Rev. B 53, 7539 (1996).

${ }^{15}$ N. S. Luo, S. Y. Wu, and C. S. Jayanthi, Phys. Rev. B 55, 11300 (1997).

${ }^{16}$ L. Haibin, Z. Hong, and W. Yinghai, Phys. Lett. A 298, 361 (2002).

${ }^{17}$ S. C. Lee and W. J. Tzeng, Phys. Rev. B 66, 184108 (2002).

${ }^{18}$ R. B. Griffiths and W. Chou, Phys. Rev. Lett. 56, 1929 (1986).

${ }^{19}$ P. Bak, Rep. Prog. Phys. 45, 587 (1982).

${ }^{20}$ A. N. Kolmogorov and V. H. Crespi, Phys. Rev. Lett. 85, 4727 (2000).

${ }^{21}$ M. Cadek, J. N. Coleman, K. P. Ryan, V. Nicolosi, G. Bister, A. Fonseca, J. B. Nagy, K. Szostak, F. Beguin, and W. J. Blau, Nano Lett. 4, 353 (2004). 